\title{
Extracorporeal Ammonia Clearance for Hyperammonemia in Critically III Patients: A Scoping Review
}

\author{
Thummaporn Naorungroj ${ }^{a, b}$ Fumitaka Yanase $^{a, c}$ Glenn M. Eastwood ${ }^{a}$ \\ Ian Baldwin ${ }^{a}$ Rinaldo Bellomo ${ }^{\text {a, }}$, e \\ aDepartment of Intensive Care, Austin Hospital, Melbourne, VIC, Australia; bepartment of Intensive Care, Faculty \\ of Medicine Siriraj Hospital, Mahidol University, Bangkok, Thailand; ' Monash University School and Public Health \\ and Preventive Medicine, ANZICS-RC, Melbourne, VIC, Australia; ${ }^{d}$ Centre for Integrated Critical Care, The University \\ of Melbourne, Melbourne, VIC, Australia; 'Data Analytics Research and Evaluation (DARE) Centre, The University of \\ Melbourne and Austin Health, Melbourne, VIC, Australia
}

\section{Keywords}

Hemodialysis · Continuous renal replacement therapy · Clearance $\cdot$ Ammonia $\cdot$ Liver failure $\cdot$ Urea cycle $\cdot$ Inborn errors of metabolism

\begin{abstract}
Introduction: Hyperammonemia is a life-threatening condition. However, clearance of ammonia via extracorporeal treatment has not been systematically evaluated. Methods: We searched EMBASE and MEDLINE databases. We included all publications reporting ammonia clearance by extracorporeal treatment in adult and pediatric patients with clearance estimated by direct dialysate ammonia measurement or calculated by formula. Two reviewers screened and extracted data independently. Results: We found 1,770 articles with 312 appropriate for assessment and 28 studies meeting eligibility criteria. Most of the studies were case reports. Hyperammonemia was typically secondary to inborn errors of metabolisms in children and to liver failure in adult patients. Ammonia clearance was most commonly reported during continuous renal replacement therapy (CRRT) and appeared to vary markedly from $<5 \mathrm{~mL} / \mathrm{min} / \mathrm{m}^{2}$ to $>250 \mathrm{~mL} / \mathrm{min} / \mathrm{m}^{2}$. When measured during intermittent hemodialysis (IHD), clearance was highest and correlated with blood flow rate
\end{abstract}

karger@karger.com

(c) 2020 S. Karger AG, Basel

www.karger.com/bpu

Karger"
$\left(R^{2}=0.853 ; p<0.001\right)$. When measured during CRRT, ammonia clearance could be substantial and correlated with effluent flow rate $\left(E F R ; R^{2}=0.584 ; p<0.001\right)$. Neither correlated with ammonia reduction. Peritoneal dialysis (PD) achieved minimal clearance, and other extracorporeal techniques were rarely studied. Conclusions: Extracorporeal ammonia clearance varies widely with sometimes implausible values. Treatment modality, blood flow, and EFR, however, appear to affect such clearance with IHD achieving the highest values, PD achieving minimal values, and CRRT achieving substantial values especially at high EFRs. The role of other techniques remains unclear. These findings can help inform practice and future studies.

(c) 2020 S. Karger AG, Basel

\section{Introduction}

Ammonia is a toxic metabolite mainly metabolized by the liver via the urea cycle [1]. Impairment of ammonia clearance causes hyperammonemia [2]. Marked hyperammonemia requires emergency management because

The protocol was registered in Open Science Framework (ID: osf.io/ dasxv). 
of its potential to induce irreversible neurological damage $[3,4]$. Immediate management aims to achieve a rapid reduction in blood ammonia levels to prevent cerebral edema and brain herniation [4]. Extracorporeal blood purification techniques have been used as a rescue therapy in this setting and have been reported as an effective adjunctive method to remove ammonia when either medications fail to do so or the level of ammonia is very high, or there is inadequate endogenous capacity to remove it from the circulation [5].

Ammonia is a small water-soluble molecule and can theoretically be removed by extracorporeal diffusionbase therapies and via standard hemofilters or dialyzers $[2,6]$. However, the optimal timing to initiate such extracorporeal treatment remains unclear and protocols vary according to center $[1,4]$. Additionally, there is a lack of data about the ability of extracorporeal therapy to achieve sufficient ammonia clearance. This is because most studies only report the time to achieve a median $50 \%$ blood ammonia level reduction rather than the direct measurement of clearance via the extracorporeal circuit [7-9]. Such reduction, however, is affected by confounders such as a gradient dependence, heterogeneity of measurement methods, calculation formulas, and other co-interventions, that is, adequate total caloric intake with protein restriction, and pharmacological therapy $[10,11]$.

Accordingly, we performed a scoping review to investigate ammonia clearance by extracorporeal treatments in hyperammonemic adult and pediatric patients and to identify potential factors affecting such clearance. We hypothesized that such ammonia clearance would vary significantly according to technique and that, during continuous renal replacement therapy (CRRT), it would be affected by the effluent flow rate (EFR).

\section{Methods}

\section{Type of Studies}

Due to lack of randomized controlled trials, we considered all publication types in the English-language articles published through to May 29, 2020. We included prospective observational studies, case reports, case series, abstracts, comments and letters to editors that had reported data on ammonia clearance. The protocol was registered in Open Science Framework (ID: osf.io/dasxv).

Types of Participants

We included patients diagnosed with hyperammonemia of any etiology and managed with extracorporeal treatment regardless of modality. We included neonates, children, and adults with hyperammonemia. Hyperammonemia was defined as blood ammonia level higher than upper limit of normal as reported in such studies irrespective of the method used to measure blood ammonia level.
Types of Interventions

Renal replacement therapy included intermittent hemodialysis (IHD), CRRT with convective and diffusive clearance or both (continuous veno-venous hemofiltration [CVVH], continuous veno-venous hemodialysis, and continuous veno-venous hemodiafiltration), and peritoneal dialysis (PD). Other extracorporeal treatments (ET) included exchange transfusion, and artificial and bioartificial liver treatment.

\section{Type of Outcome Measurements}

We calculated ammonia clearance from direct measurements of ammonia levels in both effluent or dialysis fluid and or blood from pre- and post-membrane using the Cordoba formula ([filter ammonia concentration $\times$ volume of filtrate]/plasma ammonia concentration $\times$ time) [6]. We also included studies that calculated indirect ammonia clearance from pre- and post-membrane ammonia level using Wiegand formula ([ammonia prefilter - ammonia postfilter] $\times$ blood flow rate/ammonia prefilter) [12].

\section{Search Methods for Identification of Studies}

The search for this scoping review was performed using EMBASE and MEDLINE electronic database. All databases were searched by incorporated Medical Subject Heading term and keywords in all fields (the final search strategies were provided in online suppl. Appendix 1; for all online suppl. material, see www. karger.com/doi/10.1159/000512100). Moreover, additional relevant articles were identified from the review of citation references.

Data Collection and Analysis

Selection of Studies

Two review authors (T.N. and F.Y.) checked the titles and abstracts to identify relevant articles. Then, 2 reviewers (T.N. and F.Y.) examined studies that potentially met the inclusion criteria and eligibility. One author (T.N.) extracted data and cross-checked all studies for accuracy against the original publications with another author (F.Y.). We resolved disagreements between the 2 review authors by discussion with a third review author (G.E. or R.B.) until consensus was reached.

\section{Data Extraction and Management}

Independent extraction of articles was by 2 authors using predefined criteria. Disagreements were resolved by discussion between the 2 authors and if no agreement could be reached, a third author was asked to assist with the final decision. The extracted data included patient characteristics, extracorporeal treatment characteristics, duration of treatment, ammonia clearance, co-intervention, and outcomes.

\section{Results}

\section{Description of Studies}

We identified 1,770 eligible references through the initial search. After reading titles and abstracts, 1,458 of these references were excluded because there were duplicates, review articles, animal studies, and in vitro experiments. Thus, 312 references were retrieved for further assessment. Of these, 284 references were excluded because 
Fig. 1. Flow diagram of study. RRT, renal

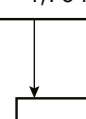
$(n=6)$

Records excluded $(n=1,026)$

Review article $(n=360)$

In vitro or animal $(n=142)$

Proceeding $(n=3)$

Non-ammonia related RRT $(n=518)$

Cannot access $(n=3)$ they did not report ammonia clearance. The remaining 28 references were included into the final review. The study flow diagram is shown in Figure 1. Neither large randomized nor interventional clinical trial of patients with hyperammonemia was found. There were 20 case reports, 2 prospective and 3 retrospective cohort studies, and 3 letters to editors.

\section{Ammonia Clearance during CRRT}

The search found 13 publications $[10,13-24]$ that reported ammonia clearance by CRRT modality. The characteristics of patients and treatment in such studies are shown in Table 1 and online suppl. Table 1. Most studies were case reports. Only 2 studies were in adult patients, and 11 studies included neonates and children. Slack et al. [10] showed that in adult liver failure patients, high volume CVVH (EFR $\sim 5,900 \mathrm{~mL} / \mathrm{h}$ ) had a significantly greater ammonia clearance compared to standard volume CVVH (EFR 2,200 mL/h). The most common causes of hyperammonemia pediatric patients was an inborn error of metabolism in the urea cycle treated in the first week of life. Both continuous arteriovenous and veno-venous therapy were used with specific techniques and prescriptions. Notably, the ammonia level before starting extracorporeal treatment were greater in pediatric patients compared to adult patients. When adjusted for body surface area, in particular, the magnitude of reported ammonia clearance varied dramatically from 4 to $275 \mathrm{~mL} / \mathrm{min} / \mathrm{m}^{2}$ and the time to $50 \%$ reduction ranged widely from 2 to $10 \mathrm{~h}$.

Ammonia clearance was increased by either higher dialysate flow rate (DFR) or ultrafiltration rate (dialysis dose), a higher blood flow rate (BFR), and removal by a longer duration of treatment. There was a moderate correlation between ammonia clearance and EFR (Fig. 2a). There were also important co-interventions which included restriction in protein intake, pharmacological treatments, and other concomitant extracorporeal treatments. Only 1 study compared 2 formulas of ammonia clearance (Cordoba and Wiegand formula) [10]. 


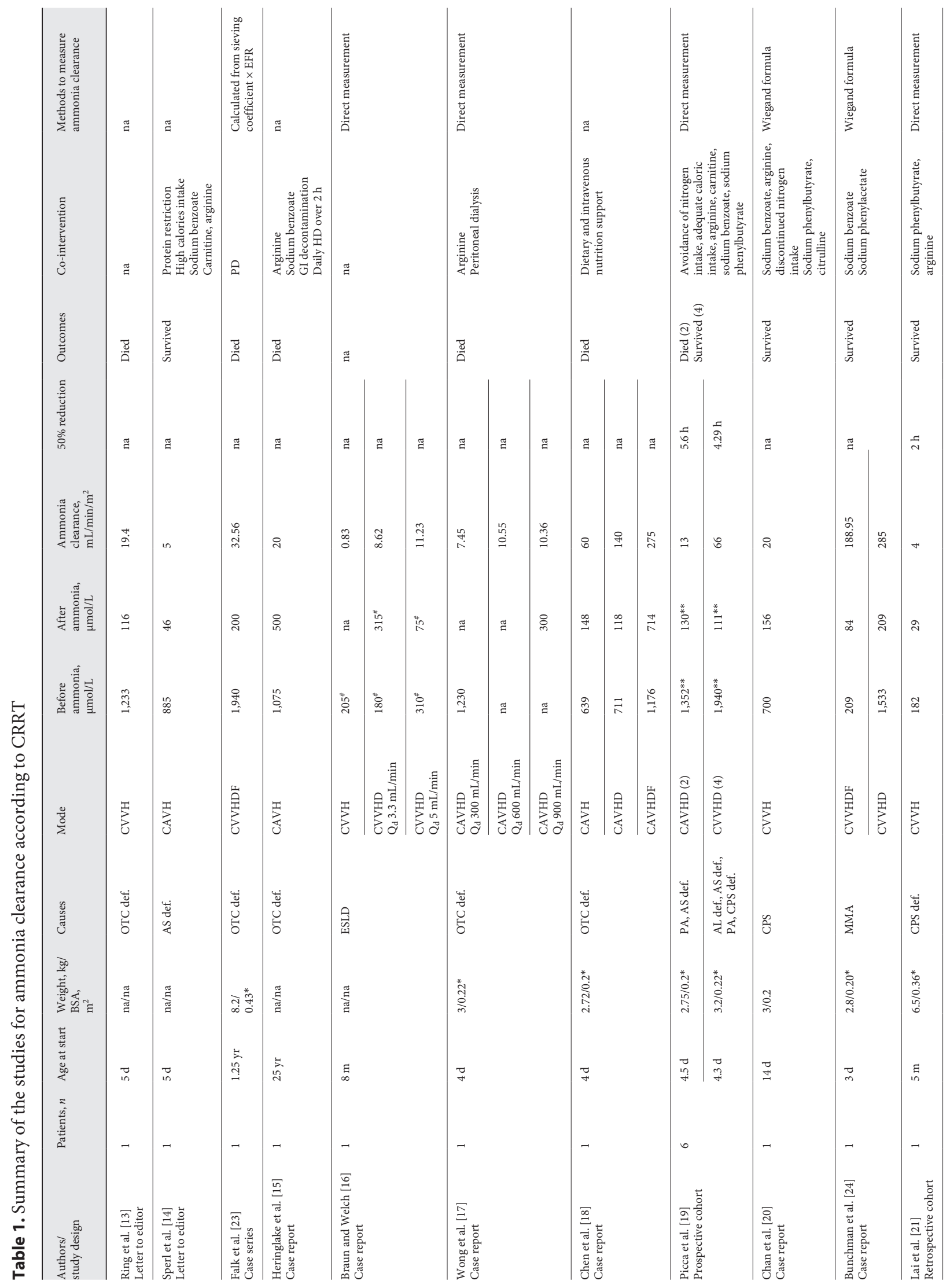




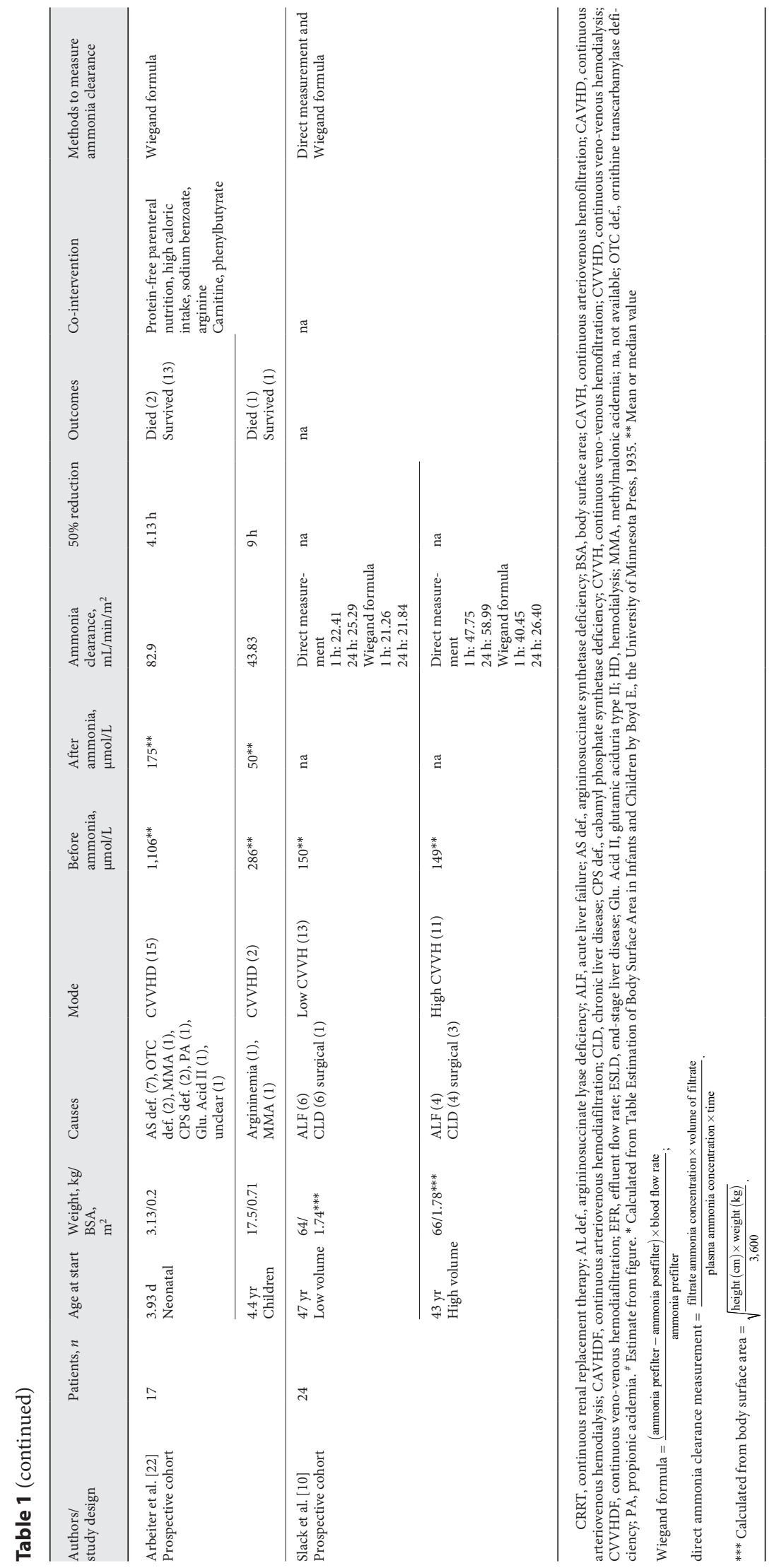


Fig. 2. Ammonia clearance according to CRRT and IHD. a Ammonia clearance according to CRRT. b Ammonia clearance according to IHD. CRRT, continuous renal replacement therapy; IHD, intermittent hemodialysis; EFR, effluent flow rate; BFR, blood flow rate.

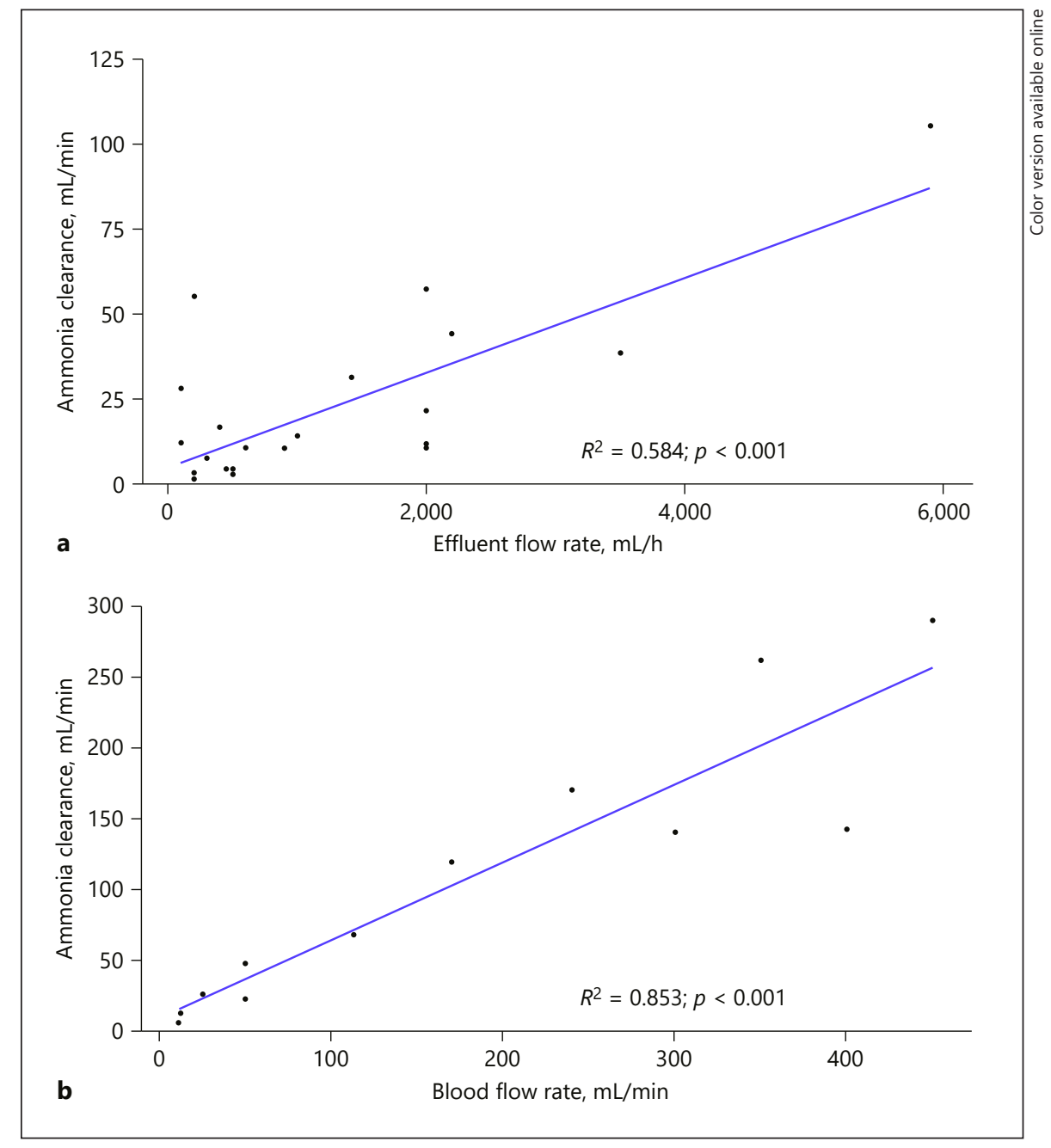

\section{Ammonia Clearance in IHD}

This search resulted in 13 publications [12, 19, 24-35] that reported ammonia clearance by IHD, and the characteristics of patients and treatment are shown in online suppl. Tables 2 and 3. Ammonia clearance was somewhat higher, and the time to $50 \%$ reduction was relatively faster than that with other modalities especially when IHD was added to an extracorporeal membrane oxygenator. Four studies also reported urea clearance but did not show a correlation between urea and ammonia clearance $[26,27,34,35]$. The correlation between ammonia clearance and BFR was robust and is shown in Figure 2b. Similar to CRRT, both protein dietary restriction and ammonia lowering agents were a common co-intervention.

\section{Ammonia Clearance in PD}

The search found 6 publications $[12,17,18,22,36,37]$ that reported ammonia clearance by $\mathrm{PD}$, and the charac- teristics of patients and treatment are shown in online suppl. Tables 4 and 5. Ammonia clearance during PD was low $(<40 \mathrm{~mL} / \mathrm{min}$ in all studies) especially in pediatric patients. Moreover, most pediatric studies reported that PD was insufficient to achieve adequate ammonia clearance. Thus, other interventions were needed to augment ammonia clearance, and PD was used as adjunctive therapy or maintenance therapy in combination with other treatment modalities.

\section{Ammonia Clearance in Other ET}

We found 2 publications using 3 different therapies $[18,38]$ reporting ammonia clearance by ET. First, Chen et al. [18] reported that ammonia clearance by blood exchange transfusion was $43 \mathrm{~mL} / \mathrm{min}$. Second, Krisper et al. [38] reported that the Prometheus system $^{\mathrm{TM}}$ had approximately twice as much ammonia clearance than the Molecular Adsorbent Recirculating 
System (141 vs. $75 \mathrm{~mL} / \mathrm{min}$, respectively). The characteristics of patients and treatment are shown in online suppl. Tables 6 and 7.

\section{Discussion}

\section{Key Findings}

We identified 28 studies and found that IHD achieved the highest ammonia clearance followed by CRRT and, at very low levels, PD. Moreover, such clearance varied widely and, sometimes, implausibly. Finally, it correlated with BFR and DFR during IHD and with EFR during CRRT.

\section{Comparing to Previous Studies}

Inborn error of metabolism cause hyperammonemia according to enzymatic defect in urea cycle or inhibition of mitochondria and acetyl coenzyme A function. On the other hand, adult liver failure initiates hyperammonemia from impaired hepatic function and portosystemic shunt and is worsened by concurrent renal impairment $[10,39]$. The ideal clearance and mass removal in these settings would have to be adjusted to the severity of hyperammonemia and the clinical condition of the patient. In this regard, ammonia can theoretically be cleared by dialytic technique because of its low molecular weight (17 Da) and minimal protein binding [34]. In an vitro study, ammonia clearance was approximately $50-80 \mathrm{~mL} / \mathrm{min}$ when BFR was $100 \mathrm{~mL} / \mathrm{min}$ [40].

However, the estimation of clearance is problematic because ammonia is an unstable molecule $[41,42]$ and its levels are affected by sample temperature [30]. This requires specimen collection, handling, and transport on ice. However, few studies have reported the details of such critical information [10,22].

Two formulas have typically been used to estimate ammonia clearance $[6,12]$. However, clearance determined by Wiegand formula is based on pre- and post-dilution ammonia concentration during CRRT. This approach has problems including post-dialysis ammonia rebound due to transport from the cellular compartment, recirculation [30, 33, 43], and the impact of replacement fluid delivery-associated hemodilution and net ultrafiltrationassociated hemoconcentration.

Theoretically, post-dilution should have a greater clearance because of a higher solute concentration in the filter. However, it may also increase filter clotting and filter downtime. Nonetheless, the impact of pre-dilution and post-dilution on daily ammonia clearance during
CRRT is unknown. Uchino et al. [44] reported that postdilution did not affect change in urea and $\mathrm{Cr}$ levels. Similarly, continuous veno-venous hemodiafiltration may be delivered with or without pre-dilution and be similarly affected. Unfortunately, no actual data exist comparing different techniques of CRRT in relation to ammonia clearance.

Finally, ammonia clearance is only one determinant of total ammonia mass removal. Ammonia mass removal mostly depends on BFR, ammonia concentration in plasma, ammonia clearance, and duration of extracorporeal treatment. Thus, even assuming a steady state and equivalent ammonia concentration in plasma (an unlikely situation with IHD), CRRT will result in greater mass ammonia removal and higher efficiency than IHD despite having a lower instantaneous clearance [45]. While IHD will have higher instantaneous clearance than CRRT, it is only applied for a short time (typically $4 \mathrm{~h}$ ). In contrast, CRRT is applied for close to $24 \mathrm{~h}$ /day. Assuming an ammonia clearance of $30-50 \%$ of urea clearance and given that urea clearance is close to EFR or dialysate flow rate in CRRT and close to BFR in IHD, some results from published studies are simply mathematically and dialytically impossible.

\section{Clinical Implications}

Our findings imply that IHD achieves the highest ammonia clearance, which, as expected, is dependent on BFR and DFR. Moreover, they imply that PD achieves inadequate clearance to control hyperammonemia. In contrast, CRRT can achieve substantial ammonia clearances, which can be regulated by increasing the EFR. Thus, they imply that because of the duration of CRRT, such therapy would result in greater ammonia mass removal over a 24 -h period than other techniques. Finally, whether other techniques such as the Prometheus ${ }^{\mathrm{TM}}$ and Molecular Adsorbent Recirculating System technique, are superior for ammonia removal remain unclear due to the lack of sufficient studies.

\section{Study Strengths and Limitations}

Our study has several strengths. It is the first to report and compare ammonia clearance among difference extracorporeal treatments. It presents a comprehensive assessment of all extracorporeal techniques in children and adults. It highlights deficiencies in our current knowledge and identified the serious limitations of PD in this context. Thus, our study provides an inclusive evaluation of ammonia clearance to inform practice and to help design further investigations. 
However, we acknowledged several limitations. First, due to lack of well-designed controlled trials and heterogeneity among studies, we performed only a scoping review without meta-analysis. Moreover, some articles are historical, and some reports used an outdated (e.g., CAVH) modality. Second, this study mainly focused on ammonia clearance and we did not have data on the adverse events or harm associated with the extracorporeal treatment of hyperammonemia. However, most studies were case reports and carried a high risk of bias. Third, the accuracy and reproducibility of ammonia measurements and the adequacy of sample collection and transport could not be evaluated. Moreover, time to specimen, recirculation, dilution and hemoconcentration effects, and the presence of a rebound phenomenon after IHD could not be assessed due to lack of reporting of such data in the analyzed studies. Thus, to a degree, our findings may represent an under-and overestimation of ammonia clearance. However, we expect that our study will promote higher quality research and measurement validation in this field in future studies.

\section{Conclusion}

Reported clearances vary widely. However, IHD achieves the highest instantaneous ammonia clearances, which are dependent on BFR and DFR. In contrast, PD achieves very low ammonia clearances. CRRT can achieve substantial ammonia clearances, which increase with higher EFR. Moreover, because of its longer daily application, CRRT may deliver greater ammonia mass removal over a 24 -h period. Finally, the role of other techniques has not be adequately explored. These findings can inform future studies targeting ammonia clearance in situations of marked hyperammonemia.

\section{Statement of Ethics}

Human research Ethics Committee review was not required for this scoping review.

\section{Conflict of Interest Statement}

Dr. Yanase reported receiving scholarship for $\mathrm{PhD}$ course in Monash University from Japan Student Services Organization and Endeavor Scholarship. Dr. Bellomo reported receiving grants from Baxter International outside the submitted work. Dr. Naorungroj, Dr. Eastwood, and Professor Balwin declare that they have no relevant financial interests.

\section{Funding Sources}

The authors have no funding sources to declare.

\section{Author Contributions}

Research idea and study design: T.N., F.Y., G.E., I.B., and R.B.; data acquisition: T.N. and F.Y.; data analysis/interpretation: all authors; supervision or mentorship: G.E., I.B., and R.B. Each author contributed important intellectual content during manuscript drafting or revision and accepts accountability for the overall work by ensuring that questions pertaining to the accuracy or integrity of any portion of the work are appropriately investigated and resolved.

\section{References}

1 Gupta S, Fenves AZ, Hootkins R. The role of RRT in hyperammonemic patients. Clin J Am Soc Nephrol. 2016;11(10):1872-8.

2 Warrillow SJ, Bellomo R. Preventing cerebral oedema in acute liver failure: the case for quadruple- $\mathrm{H}$ therapy. Anaesth Intensive Care. 2014;42(1):78-88.

3 Redant S, Beretta-Piccoli X, Mugisha A, Attou R, Kaefer K, De Bels D, et al. Hyperammonemia, the last indication of high-volume hemodiafiltration in adult and children: a structured review. Blood Purif. 2019;48(4):330-5.

4 Raina R, Bedoyan JK, Lichter-Konecki U, Jouvet $\mathrm{P}$, Picca S, Mew NA, et al. Consensus guidelines for management of hyperammonaemia in paediatric patients receiving continuous kidney replacement therapy. Nat Rev Nephrol. 2020;16(8):471-82.
5 El-Hattab AW. Inborn errors of metabolism. Clin Perinatol. 2015;42(2):413-39.

6 Cordoba J, Blei AT, Mujais S. Determinants of ammonia clearance by hemodialysis. Artif Organs. 1996;20(7):800-3.

7 Schaefer F, Straube E, Oh J, Mehls O, Mayatepek E. Dialysis in neonates with inborn errors of metabolism. Nephrol Dial Transplant. 1999;14(4):910-8.

8 Rajpoot DK, Gargus JJ. Acute hemodialysis for hyperammonemia in small neonates. Pediatr Nephrol. 2004;19(4):390-5.

9 Westrope C, Morris K, Burford D, Morrison G. Continuous hemofiltration in the control of neonatal hyperammonemia: a 10-year experience. Pediatr Nephrol. 2010;25(9):172530.
10 Slack AJ, Auzinger G, Willars C, Dew T, Musto $\mathrm{R}$, Corsilli $\mathrm{D}$, et al. Ammonia clearance with haemofiltration in adults with liver disease. Liver Int. 2014;34(1):42-8.

11 Liu J, Lkhagva E, Chung H-J, Kim H-J, Hong S-T. The pharmabiotic approach to treat hyperammonemia. Nutrients. 2018; 10(2): 140 .

12 Wiegand C, Thompson T, Bock GH, Mathis RK, Kjellstrand CM, Mauer SM. The management of life-threatening hyperammonemia: a comparison of several therapeutic modalities. J Pediatr. 1980;96(1):142-4.

13 Ring E, Zobel G, Stöckler S. Clearance of toxic metabolites during therapy for inborn errors of metabolism. J Pediatr. 1990;117(2 Pt 1):349-50. 
14 Sperl W, Geiger R, Maurer H, Guggenbichler JP. Continuous arteriovenous haemofiltration in hyperammonaemia of newborn babies. Lancet. 1990;336(8724):1192-3.

15 Heringlake S, Böker K, Manns M. Fatal clinical course of ornithine transcarbamylase deficiency in an adult heterozygous female patient. Digestion. 1997;58(1):83-6.

16 Braun MC, Welch TR. Continuous venovenous hemodiafiltration in the treatment of acute hyperammonemia. Am J Nephrol. 1998;18(6):531-3.

17 Wong KY, Wong SN, Lam SY, Tam S, Tsoi NS. Ammonia clearance by peritoneal dialysis and continuous arteriovenous hemodiafiltration. Pediatr Nephrol. 1998;12(7):589-91.

18 Chen CY, Chen YC, Fang JT, Huang CC. Continuous arteriovenous hemodiafiltration in the acute treatment of hyperammonaemia due to ornithine transcarbamylase deficiency. Ren Fail. 2000;22(6):823-36.

19 Picca S, Dionisi-Vici C, Abeni D, Pastore A Rizzo C, Orzalesi M, et al. Extracorporeal dialysis in neonatal hyperammonemia: modalities and prognostic indicators. Pediatr Nephrol. 2001;16(11):862-7.

20 Chan WK, But WM, Law CW. Ammonia detoxification by continuous venovenous haemofiltration in an infant with urea cycle defect. Hong Kong Med J. 2002;8(3):207-10.

21 Lai YC, Huang HP, Tsai IJ, Tsau YK. Highvolume continuous venovenous hemofiltration as an effective therapy for acute management of inborn errors of metabolism in young children. Blood Purif. 2007;25(4):303-8.

22 Arbeiter AK, Kranz B, Wingen AM, Bonzel KE, Dohna-Schwake C, Hanssler L, et al. Continuous venovenous haemodialysis (CVVHD) and continuous peritoneal dialysis (CPD) in the acute management of 21 children with inborn errors of metabolism. Nephrol Dial Transplant. 2010;25(4):125765.

23 Falk MC, Knight JF, Roy LP, Wilcken B, Schell DN, O'Connell AJ, et al. Continuous venovenous haemofiltration in the acute treatment of inborn errors of metabolism. Pediatr Nephrol. 1994;8(3):330-3.
24 Bunchman TE, Barletta GM, Winters JW, Gardner JJ, Crumb TL, McBryde KD. Phenylacetate and benzoate clearance in a hyperammonemic infant on sequential hemodialysis and hemofiltration. Pediatr Nephrol. 2007; 22(7):1062-5.

25 Kiley JE, Pender JC, Welch HF, Welch CS. Ammonia intoxication treated by hemodialysis. N Engl J Med. 1958;259(24):1156-61.

26 Kaneda H, Haruyama T, Chiba S, Sugai Y, Hirasawa $\mathrm{T}$, Takeuchi $\mathrm{M}$, et al. A patient with recurrent hepatic encephalopathy and chronic renal failure treated successfully with longterm hemodialysis. Tohoku J Exp Med. 1977; 123(3):227-34.

27 Watson AJ, Chambers T, Karp JE, Risch VR, Walker WG, Brusilow SW. Transient idiopathic hyperammonaemia in adults. Lancet. 1985;2(8467):1271-4.

28 Rutledge SL, Havens PL, Haymond MW, McLean RH, Kan JS, Brusilow SW. Neonatal hemodialysis: effective therapy for the encephalopathy of inborn errors of metabolism. J Pediatr. 1990;116(1):125-8.

29 Tuchman M, Mauer SM, Holzknecht RA, Summar ML, Vnencak-Jones CL. Prospective versus clinical diagnosis and therapy of acute neonatal hyperammonaemia in two sisters with carbamyl phosphate synthetase deficiency. J Inherit Metab Dis. 1992;15(2):269-77.

30 Pimentel JL Jr, Brusilow SW, Mitch WE. Unexpected encephalopathy in chronic renal failure: hyperammonemia complicating acute peritonitis. J Am Soc Nephrol. 1994; 5(4):1066-73.

31 Elshihabi I, Brzowski A, Kaye C, Kearon P. Efficiency of hemodialysis therapy for a urea cycle defect in a neonate. Clin Nephrol. 1995; 43(3):208-9.

32 Summar M, Pietsch J, Deshpande J, Schulman G. Effective hemodialysis and hemofiltration driven by an extracorporeal membrane oxygenation pump in infants with hyperammonemia. J Pediatr. 1996;128(3):379-82.

33 Vats A, Kashtan CE, Tuchman M, Mauer M. Hemodialysis catheter placement and recirculation in treatment of hyperammonemia. Pediatr Nephrol. 1998;12(7):592-5.
34 Levesque R, Leblanc M, Cardinal J, Teitlebaum J, Skrobik Y, Lebrun M. Haemodialysis for severe hyperammonaemic coma complicating urinary diversions. Nephrol Dial Transplant. 1999;14:458-61.

35 Lévesque R, Cardinal J, Leblanc M. Haemodialysis for hyperammonaemic encephalopathy. Nephrol Dial Transplant. 2000;15(7): 1101.

36 Siegel NJ, Brown RS. Peritoneal clearance of ammonia and creatinine in a neonate. J Pediatr. 1973;82(6):1044-6.

37 Pipili C, Polydorou A, Pantelias K, Korfiatis P, Nikolakopoulos F, Grapsa E. Improvement of hepatic encephalopathy by application of peritoneal dialysis in a patient with non-endstage renal disease. Perit Dial Int. 2013;33(2): 213-6.

38 Krisper P, Haditsch B, Stauber R, Jung A, Stadlbauer $\mathrm{V}$, Trauner $\mathrm{M}$, et al. In vivo quantification of liver dialysis: comparison of albumin dialysis and fractionated plasma separation. J Hepatol. 2005;43(3):451-7.

39 Savy N, Brossier D, Brunel-Guitton C, Ducharme-Crevier L, Du Pont-Thibodeau G, Jouvet P. Acute pediatric hyperammonemia: current diagnosis and management strategies. Hepat Med. 2018;10:105-15.

40 Kiley JE, Welch HF, Pender JC, Welch CS. Removal of blood ammonia by hemodialysis. Proc Soc Exp Biol Med. 1956;91(3):489-90.

41 Huizenga JR, Tangerman A, Gips CH. Determination of ammonia in biological fluids. Ann Clin Biochem. 1994;31(Pt 6):529-43.

42 Hester JR, Korzun WJ, Mabry LU. Blood ammonia stability revisited. Clin Lab Sci. 2015; 28(3): 173-7.

43 Hemodialysis Adequacy 2006 Work Group. Clinical practice guidelines for hemodialysis adequacy, update 2006. Am J Kidney Dis 2006;48(Suppl 1):S2-90.

44 Uchino S, Fealy N, Baldwin I, Morimatsu H, Bellomo R. Pre-dilution vs. post-dilution during continuous veno-venous hemofiltration: impact on filter life and azotemic control. Nephron Clin Pract. 2003;94(4):c94-8.

45 Clark WR, Henderson LW. Renal versus continuous versus intermittent therapies for removal of uremic toxins. Kidney Int Suppl. 2001;78:S298-303. 